\title{
LA INFANCIA DE MAYA ANGELOU EN EL SUR DE ESTADOS UNIDOS Y SU EXILIO VOLUNTARIO
}

\author{
THE CHILDHOOD OF MAYA ANGELOU IN SOUTH UNITED STATES AND HER \\ VOLUNTARY EXILE
}

Mª Eugenia Sánchez Suárez

UNED de Madrid

\section{RESUMEN:}

En este artículo analizaremos la figura de Maya Angelou, escritora norteamericana. Con la ayuda de su obra autobiográfica nos centraremos en dos etapas importantes: su infancia y su exilio, períodos de la vida de Maya llenos de prejuicios y dificultades frutos del racismo de la época.

\section{PALABRAS ClAVES:}

Maya Angelou, exilio, prejuicio racial.

\section{ABSTRACT:}

This article aims to analyse the figure of Maya Angelou, American writer. With the help of her autobiographic work, we will focus on two important periods: her childhood and her exile, when Maya suffered from the prejudices and the difficulties as a result of the racism in those days.

\section{KEY WORD:}

Maya Angelou, exile, racial prejudice. 
Son múltiples las barreras físicas, mentales y sociales que Maya Angelou ha contribuido a socavar mediante su obra y su activismo político. El que haya conseguido superar tantos obstáculos pese a haber sido víctima de la segregación racial, el sexismo e incluso la pederastia se explica por su valor, en su opinión, la virtud más importante, porque sin él es imposible poner en práctica las demás virtudes de una manera consistente (08-10-07). La vida de esta autora ha girado, pues, en torno a la supervivencia, pero no entendida como "just bare, awful, plodding survival" sino como "survival with grace and faith" (cit. en McPherson, 1991: 10-11)

La obra de Angelou se inscribe en una tradición de escritores y escritoras de color para quienes la autobiografía ha supuesto un foro abierto en el que exponer los detalles de su lucha, informar al lector sobre la triste condición de su raza y promover un movimiento de protesta que facilitase la consecución de la plena igualdad. En este sentido, no hemos de olvidar la influencia política que ha ejercido Angelou, no sólo por haber sido colaboradora directa de Martin Luther King o de Malcolm X, sino porque su obra ha sido capaz de "encarnar" el sistema ideológico de la segregación racial hasta en sus más nimios detalles, reconstituyendo no sólo su contexto exterior sino, lo que es más importante, el interior, el vivencial. Con ello ha contribuido sin duda a proclamar nuevos valores de civilización y convivencia. Otra importante aportación de Angelou a la tradición literaria afroamericana consiste en haber desafiado los estereotipos tradicionales con los que se representaba a la mujer de color y haber celebrado, a través de las figuras de su abuela, Momma Henderson, de su madre, Vivian Baxter, y finalmente de ella misma, "the unsung spirit of noncompliant Black Women" (O'Neale 1984: 31). Hemos de tener en cuenta, a este respecto, que la mujer aparece en la literatura en general como símbolo antes que persona. Su figura ha sido siempre más difícil de conceptualizar que la del hombre y particularmente susceptible de asociar a las más diversas representaciones, imágenes, mitos, etc. El que Angelou haya introducido una mayor diversidad en la forma de representar a la mujer de color, y en especial el que la haya representado como matriarca hábil y poderosa, ha contribuido, pues, a superar prejuicios raciales y sexistas. Así, por ejemplo, la acusada "movilidad" que se advierte en la propia vida de la escritora constituye una nueva cualidad en una mujer de color, tradicionalmente restringida a un espacio muy limitado a causa de su raza y de las funciones sociales que le asignaban como mujer (Christian, 1985: 182)

Nos referiremos a continuación a la obra de Maya Angelou mediante el estudio de tres de sus autobiografías. En I Know Why the Caged Bird Sings (1969), su obra más conocida y más aclamada por la crítica, se nos narra su infancia en el sur de los EEUU en tiempos de la Depresión. The Heart of a Woman (1981) recoge su experiencia como activista política en las décadas de los cincuenta y los sesenta. Finalmente, en
All God's Children Should Have Travelling Shoes (1986) se nos narra su experiencia cuando, sintiéndose decepcionada por la falta de resultados obtenidos en la lucha por los derechos civiles y, tras el fracaso de una relación sentimental que la lleva a África la escritora decide exiliarse en Ghana de manera voluntaria.

\section{UNA INFANCIA EN EL SUR}

En I Know Why the Caged Bird Sings (1969), Maya (nombre que Angelou le debe a su hermano Bailey por la forma en la que éste pronunciaba "My sister") se nos presenta como una niña que adquiere pronto conciencia de "outsider" debido a su color, a su carácter retraído y soñador, y a una peculiar situación familiar en la que sus padres, divorciados, parecen haberse desentendido de sus hijos. Los niños son enviados a Stamps (Arkansas), donde vive su abuela paterna, Momma, un lugar donde la segregación racial es tan radical que "most Black children didn't really, absolutely know what whites looked like" (Angelou, 1969: 24-25). Como excepción tenemos a las niñas "powhitetrash", la "basura blanca", a quienes la pobreza obliga a vivir entre los negros, y que protagonizan un "duelo" inolvidable con Momma, una de esas matriarcas afroamericanas que aportan la estabilidad en familias en las que los hombres entran y salen. En un momento determinado las niñas blancas convierten a Momma en blanco de sus bromas, buscando una reacción agresiva que habría podido tener consecuencias trágicas tanto para ella como para su familia. La abuela, para dolor y frustración de Maya, que observa todo en silencio, se mantiene firme, aparentemente impasible, mientras sonríe y tararea un himno religioso. A pesar de romper a llorar al ver las humillaciones que ha de soportar su abuela, Maya se da cuenta finalmente de que "Whatever the contest had been out front, I knew Momma had won" (Angelou, 1969: 32). Ésta ha demostrado su habilidad para sobrevivir en un medio extremadamente hostil.

Con sus ojos de niña, esperando poder escapar de "my black ugly dream" (Angelou, 1969: 4), Maya explora la naturaleza y la tragedia de la opresión racial hasta en los más nimios detalles de la vida cotidiana. Así, por ejemplo, una mujer para la que trabaja un tiempo como empleada doméstica, Mrs Cullinan, se cree con derecho a cambiar los nombres de sus empleadas de color; Hallelujah recibe el nombre de Miss Glory, y Marguerite (Maya) el de Mary. Maya se rebela entonces por primera vez ante este intento de anular su identidad y fuerza su despido rompiendo unas valiosas piezas de cristalería. Otro episodio significativo en la vida de Maya es su violación a manos de Mr Freeman, que convive con su madre en St. Louis, donde la niña y su hermano Bailey pasan una temporada. Tras descubrirse lo sucedido, Maya ha de testificar en un juicio y miente por no decepcionar a los suyos, afirmando que Mr Freeman no había mostrado interés sexual por ella anteriormente; por ello, cuando su violador aparece muerto, 
Maya se siente en cierta medida responsable y se asusta del poder potencial de la palabra: "a man was dead because I lied" (Angelou, 1969: 84). Decide entonces dejar de hablar, salvo con Bailey. Esta imagen de una niña muda como consecuencia de una tragedia resulta particularmente representativa de un pueblo silenciado por la esclavitud. A Maya la saca de esa postración la "aristócrata" de los negros de Stamps, Mrs. Bertha Flowers, una mujer culta e independiente económicamente que la hace entender que merece respeto y atención: "It would be safe to say that she made me proud to be Negro, just by being herself" (Angelou, 1969: 92). Mrs Flowers la anima a leer en voz alta y le muestra cómo hacerlo con A Tale of Two Cities. Maya comenta: "I heard poetry for the first time in my life" (Angelou, 1969: 97). Un aspecto fundamental en I Know Why the Caged Bird Sings es la existencia de la comunidad negra que, a modo de familia extensa, nutre a sus miembros y los ayuda a sobrevivir frente a la pobreza y el prejuicio racial. Los lazos que se establecen se ven reforzados gracias a la iglesia negra, prácticamente el único espacio en el que los afroamericanos pueden desarrollar un cierto tipo de activismo. La Colored Methodist Episcopal Church de Stamps promueve un código rígido de comportamiento, como observamos en Momma, pero constituye también una fuente de conocimiento y de orgullo, dado que a los negros les corresponde, en calidad de pueblo oprimido, la tierra prometida. Por otra parte, Maya se enamora por primera vez de la palabra a través de la lectura de la Biblia y de su asistencia a los servicios religiosos:

Deuteronomy was my favorite book in the Bible. The laws were so absolute, so clearly set down, that I knew if a person truly wanted to avoid hell and brimstone, and being roasted forever in the devil's fire, all she had to do was memorize Deuteronomy and follow its teaching, word for word. I also liked the way the word rolled off the tongue (Angelou, 1969: 38).

Además de lo expuesto, dos episodios resultan particularmente significativos en I Know Why the Caged Bird Sings, en el sentido de que le permiten a Maya aprender una serie de verdades sobre sí misma, su comunidad y el modo en que está organizado el mundo. El primero se produce cuando Maya tiene doce años, el día de su graduación en Lafayette County Training School, un colegio reservado a los negros. Un blanco, Mr Edward Donleavy, se dirige a los estudiantes y sus familias, e insulta su inteligencia; de sus palabras se deduce que lo máximo que se espera de ellos es que algunos de los estudiantes varones sean buenos deportistas; Maya toma así conciencia de que el sistema educativo es un fiasco para los de su raza: "to my left and right the proud graduating class of 1940 had dropped their heads" (Angelou, 1969: 175). Es entonces cuando un estudiante modélico, Henry Reed, mantiene viva la llama de la esperanza cantando el llamado "himno nacional negro" de James Weldon Johnson. Este episodio da pie a Angelou para subrayar el papel que han jugado los "poetas" a la hora de sostener a la comunidad negra en su lucha, algo que podría aplicarse a su propia obra: "Oh, Black known and unknown poets, how often have your auctioned pains sustained us? Who will compute the lonely nights made less lonely by your songs, or the empty pots made less tragic by your tales?" (Angelou, 1969: 180).

Se produce otro momento de revelación cuando Momma lleva a Maya a la consulta de un dentista blanco al que había prestado dinero en tiempos de la Depresión. A la niña le corresponde ser tratada por un dentista negro, pero el más cercano vive en la lejana Texarcana y el dolor que sufre Maya es muy intenso, por lo que Momma, dadas las circunstancias, cree poder pedirle al dentista blanco un favor. Éste pronuncia entonces la siguiente infamia: “Annie, my policy is I'd rather stick my hand in a dog's mouth than in a nigger's" (Angelou, 1969: 184). A pesar, pues, de haber aceptado dinero de Momma para salvar su negocio, la deshumaniza a ella y a su nieta negando sus cualidades humanas (Lupton, 1998: 68)1․ Mientras ambas se dirigen en autobús a Texarcana, la poco expresiva Momma "put her arm around me, which was very unusual for Momma to do" (Angelou, 1969: 187).

Por amor a sus nietos, Momma decide alejarlos de Stamps el día en que obligan a Bailey a llevar el cadáver de una persona de color al interior de una cárcel y ve el odio reflejado en las caras de los prisioneros blancos. Al llegar a casa, hace una pregunta muy peligrosa en la Arkansas de 1940: "Uncle Willie, why do they hate us so much?" (Angelou, 1969: 192). Con el fin de protegerle, tanto Momma como Willy le responden con evasivas: "Would Momma, who knew the ways of the whites and the wiles of the Blacks, try to answer her grandson, whose very life depended on his not truly understanding the enigma? Most assuredly not" (Angelou, 1969: 193-4). Maya y Bailey son enviados a San Francisco con su madre, Vivian, y empiezan a perder sus miedos y a gozar de una mayor libertad. Con el firme apoyo de su madre, Maya logrará entonces transgredir algunas fronteras sociales, como ser la primera mujer negra en trabajar en los tranvías de San Francisco como conductorette; poco después, sin embargo, un embarazo temprano la lleva a abandonar su trabajo y los estudios.

\section{UNA ETAPA DE ACTIVISMO POLÍTICO}

En The Heart of a Woman (1981) se nos narran las experiencias de Maya en Nueva York como activista política a finales de los años cincuenta y principios de los sesenta un activismo que compagina con sus primeros éxitos como cantante y con su entrada en The Harlem Writers Guild, donde conoce a escritores como James Baldwin, su mentor. A pesar de los éxitos que va logrando a nivel profesional, la vida de Maya

1 Esta misma estrategia la encontramos, por ejemplo, en The Pearl de John Steinbeck cuando el doctor blanco se niega a tratar a un bebé indio y le dice a su criado: "Have I nothing better to do than cure insect bites for 'little Indians'? I am a doctor, not a veterinary" (Steinbeck, 2000: 15). 
se politiza hasta tal punto en una etapa particularmente convulsa de la historia de Estados Unidos, que decide abandonar las distintas facetas de su vocación artística. A raíz de sus actividades políticas, Maya contacta personalmente con Martin Luther King y Malcolm X, al que se siente, de hecho, más próxima porque su forma de abordar la lucha racial le parece más efectiva. Paralelamente, los problemas de su hijo Guy en el colegio demuestran hasta qué punto el odio racial corrompe todo e impide el entendimiento y la colaboración entre Maya y la directiva blanca:

If I had an eternity and the poetry of old spirituals, I could not make them live with me the painful moments when I tried to prove to Guy that his colour was not a cruel joke, but a healthful design [...] The radio and posters, newspapers and teachers, bus drivers and salespersons told him every day in thousands of ways that he had come from nothing and was going nowhere (Angelou, 1981: 19-20).

Tras decidirse a colaborar con la Southern Christian Leadership Conference (SCLC), en donde tiene que intentar superar sus propios prejuicios raciales al encontrarse con colaboradores blancos tales como Jack Murray, Angelou conoce a Luther King, "the best we had, the brightest and most beautiful" (Angelou, 1981: 55). La honestidad de la escritora no le permite, sin embargo, mitificarlo en exceso: "I looked at him and thought about the good-looking sexy school athlete, who was invariably the boyfriend of the high-yellow cheerleader" (Angelou, 1981: 93).

Maya se esfuerza por conjugar su activismo político con sus responsabilidades como madre, y se ve obligada a enfrentarse con el líder de una pandilla de jóvenes chicos de color, los Savages, que amenazan a su hijo Guy. No puede, sin embargo, evitar empatizar con dichos jóvenes: Whites, who ruled the world, owned the air and food and jobs and schools and fair play, had refused to share with them any of life's necessities -and somewhere, deeper than their consciousness, they believed the whites were correct. They, the black youth, young lords of nothing, were born without value and would creep, like blinded moles, their lives long in the darkness, under the earth, chewing on roots, driven far from the light (Angelou, 1981: 81). Tras conocer al sudafricano Vusumzi Make, representante del congreso panafricano, Maya deja su trabajo como coordinadora en la SCLC y como cofundadora de la CAWAH (The Cultural Association for Women of African Heritage) y asume el papel de la perfecta y sumisa ama de casa que Make desea que sea; sin embargo, será el propio Make quien finalmente la anime a aceptar el papel de reina negra en The Blacks de Genet. Amplía, además, sus horizontes advirtiéndole que no caiga en lo que denomina "reverse racism", intentando hacerle entender que los negros sólo son mejores porque no detentan el poder: "my dear wife, most black revolutionaries, most black activists, do not really want change. They want exchange" (Angelou, 1981: 175). Tras encontrarse con graves dificultades económicas por culpa de Make, Angelou abandona su puesto en la SCLC y viaja a Egipto junto a éste y su hijo Guy, donde parece iniciarse una nueva vida que promete hacerle olvidar su marginación anterior: "Opulent fabrics, exotic perfumes and the service of personal servants threatened to tear from my mind every memory of growing up in America as a second-class citizen" (Angelou, 1981: 216). A pesar de los prejuicios en contra de las mujeres, Maya consigue encontrar un trabajo como periodista en The Arab Observer. Tras un nuevo fracaso sentimental a causa de las infidelidades de Make, y dado que percibe hostilidad hacia ella en su entorno y en su lugar de trabajo, Maya decide establecerse en África Occidental y matricular a su hijo Guy en la Universidad de Ghana. Ha llegado a la conclusión de que África es el único lugar del mundo donde podrá librarse definitivamente del estigma de la negritud. Deja, pues, atrás su tierra de modo voluntario y se adentra en el terreno de lo desconocido. Su cuestionamiento de los márgenes de la discriminación racial la lleva a explorar un nuevo terreno, el de la dicotomía existente entre el "centro" y la "periferia", entre los que encontrará interconexiones insospechadas. Recién llegada a Ghana, un país que acaba de conseguir la independencia bajo la presidencia de Kwame Nkrumah, Maya y su hijo se quedan gratamente sorprendidos al ver pilotos de color (algo impensable en EEUU en 1962) y ser testigos de cómo los de su raza se desenvuelven con eficacia en todo tipo de posiciones de liderazgo. Parece iniciarse, pues, una etapa prometedora en sus vidas, pero un grave accidente de Guy hace temer a Maya por su vida y la obliga a cambiar de planes.

\section{EL EXILIO VOLUNTARIO}

La experiencia de Maya Angelou como mujer apátrida y emigrada se nos narra en All God's Children Should Have Travelling Shoes (1986). Por primera vez en su vida el color de su piel es el correcto, el normal, pero pronto se pone de manifiesto la ingenuidad de los "expats" (la "diáspora" negra), entre quienes se cuentan Maya y su hijo Guy. Aproximadamente doscientos afroamericanos han llegado a Ghana atraídos por la invitación del presidente Nkrumah a la población de color de todo el mundo para que se establecieran en el país y desarrollaran sus talentos bajo un gobierno progresista y revolucionario (Williams, 1997: 24). Todos los que llegan confiaban en volver al "hogar", revirtiendo el orden de la historia, pero tropiezan con la profunda desconfianza de la población de Ghana hacia ellos, que no los reconoce como sus "hijos". Sin embargo, tardan en confesarse a sí mismos la realidad: "We had come home, and if home was not what we had expected, never mind, our need for belonging allowed us to ignore the obvious and to create real places or even illusory places, befitting our imagination" (Angelou, 1986: 19).

Por otra parte, Maya no consigue borrar de su mente la posible culpabilidad de los antepasados africanos en la tragedia de los suyos: "the ugly suspicion that my ancestors 
had been weak and gullible and were sold into bondage by a stronger and more clever tribe" (Angelou, 1986: 47). A pesar de ser consciente de que existe una barrera histórica y cultural difícil de franquear entre los africanos y los "expats" americanos, que no pueden evitar rezumar "a barbarous history sewn to our dark skins" (Angelou, 1986: 76), Maya se esfuerza por integrarse en su nuevo país aprendiendo, por ejemplo, el fanti. El pequeño grupo de "expats" reacciona con una cierta ambigüedad cuando llega a Ghana la noticia de que Martin Luther King está organizando una marcha pacífica en Washington. Angelou no confía ya en la efectividad de sus métodos:

After all, when I worked for him, I had been deluded into agreeing with Reverend
King that love would cure America of its pathological illnesses, that indeed our
struggle for equal rights would redeem the country's baleful history. But all the
prayers, sit-ins, sacrifices, jail sentences, humiliation, insults and jibes had not borne out Reverend King's vision (Angelou, 1986: 122).

A pesar de ello, Maya participa en la organización de una marcha ante la embajada norteamericana. Mientras ella y sus compañeros observan a un soldado negro recoger la bandera de EEUU con sumo cuidado, alguien grita: "Why you, brother? What has that flag done for you?" (Angelou, 1986: 126). Sin embargo, en el fondo, los "expats" no pueden evitar el impulso de venerarla. Maya vuelve a contactar en África con Malcolm X, al que describe como “America's Molotov cocktail, thrown upon the White hope that all Black Americans would follow the nonviolent tenets of Dr Martin Luther King" (Angelou, 1986: 129). Malcolm, sin embargo, ha experimentado una conversión personal en La Meca, y se muestra pragmático y conciliador. Como le dice a Maya, "We need people on each level to fight our battle" (Angelou, 1986: 145). Maya explora diversas zonas de Ghana y advierte similitudes insospechadas entre la hospitalidad de la que es objeto y la solidaridad entre vecinos de la que fue testigo en el sur de los EEUU durante su infancia (Angelou, 1986: 103). Sin embargo, las diferencias culturales con los naturales de Ghana son marcadas, especialmente en el espíritu de lucha de la minoría de color norteamericana, que ha sufrido la tragedia de la esclavitud y el racismo: "Over centuries of opresion we had developed a doctrine of resistance which included false docility and sarcasm. We also had a most un-African trait: we were nearly always ready and willing to fight" (Angelou, 1986: 158). En definitiva, los afroamericanos no pueden "volver" a África. Consecuentemente, Maya decide volver a Estados Unidos para colaborar con Malcolm en la Organization of Afro American Unity (OAAU), un proyecto que no llegará a convertirse en realidad a causa del asesinato del líder. Antes de partir en 1965, Maya visita la zona de Keta, muy castigada por el fenómeno de la esclavitud. Tras advertir detalles que le resultan extrañamente familiares, una mujer la "reconoce" en el mercado e inicia una ceremonia de "duelo" por los familiares perdidos. Maya se une a su llanto, convencida de que están, efectivamente, emparentadas; a pesar de todo, extrae una lección positiva de la historia: "Despite the murders, rapes and suicides, we had survived" (Angelou, 1986: 207). Todas estas experiencias hacen madurar a la escritora, que aprende, entre otras cosas, que una actitud permanentemente belicista no va a llevarle a conseguir el tipo de sociedad que añora, lo que hace de su autobiografía seriada lo que podríamos considerar un Bildungsroman. Por otra parte, la experiencia africana marca profundamente a Angelou, que resume así el efecto que tuvo en ella: "I never felt I belonged anywhere until I went to Ghana. Then parts of me relaxed that I didn't even know I had. My soul relaxed" (Blum, cit. en Williams, 1997: 46).

A modo de conclusión, Angelou ha transformado, a través del género de la autobiografía, una experiencia privada y única en colectiva y universal; como ella misma afirma, "I see everybody's work as an example of the particular, which is indicative of the general" (Tate, 1985: 6). Siguiendo la estela iniciada por Philis Wheatley, forma parte de ese grupo de escritoras de color que han abandonado la marginalidad para establecerse como protagonistas de su tradición literaria (Christian, 1985: 28). Por otra parte, la determinación de Angelou a la hora de superar tanto el exilio interno al que la condenó el sistema ideológico de la segregación como el exilio externo que la llevó hasta Ghana la han convertido en un icono de la comunidad afroamericana.

\section{REFERENCIAS BIBLIOGRÁFICAS}

Angelou, M., I Know Why the Caged Bird Sing, Nueva York, Random House, 1969

----, The Heart of a Woman, Londres / Nueva York, Bantam Books, 1982

-.--, All God's Children Should Have Travelling Shoes, Nueva York, Random House, 1986. ,--- 08-10-07

Blum, W.,"Listening to Maya Angelou", San Francisco Examiner, 1975, pp.12-23, en Williams (ed.) Readings on Maya Angelou, San Diego, Greenhaven Press, 1997, pp. 44-50.

Chritian, B., Black Feminist Criticism: Perspectives on Black Women Writers, Nueva York / Oxford, Pergamon Press, 1985.

Evans, M. (ed.), Black Women Writers 1950-1980: A Critical Evaluation, Nueva York / Londres, Anchor Books, 1984.

Lupton, M. J., Maya Angelou: A Critical Companion, Londres, Greenwood Press, 1998.

Mcpherson, D. A., Order Out of Chaos: The Autobiographical Works of Maya Angelou, Londres, Virago, 1991.

O'Neale, S., "Reconstruction of the Composite Self: New Images of Black Women in Maya Angelou's Continuing Autobiography", en Evans (ed.), Black Women Writers 1950-1980: A Critical Evaluation Nueva York / Londres, Anchor Books, 1984, pp. 25-36.

Tate, C. (ed.), Black Women Writers at Work, Harpenden, Oldcastle Books, 1985. 
Steinbeck, J., The Pearl, Penguin Classics, 2000.

Williams, M. (ed.), Readings on Maya Angelou, San Diego, Greenhaven Press, 1997. 